\section{Cahiers de Narratologie}

Analyse et théorie narratives

$22 \mid 2012$

Voix off et narration cinématographique. Second volet

\title{
La voie de l'a-féminité
}

Voix-off et subjectivité féminine dans The Man Who Envied Women d'Yvonne Rainer

Johanna Renard

\section{(2) OpenEdition}

Édition électronique

URL : http://journals.openedition.org/narratologie/6530

DOI : $10.4000 /$ narratologie. 6530

ISSN : 1765-307X

Éditeur

LIRCES

Référence électronique

Johanna Renard, "La voie de l'a-féminité », Cahiers de Narratologie [En ligne], 22 | 2012, mis en ligne le 20 juillet 2012, consulté le 20 avril 2019. URL : http://journals.openedition.org/narratologie/6530 ;

DOI : $10.4000 /$ narratologie. 6530

Ce document a été généré automatiquement le 20 avril 2019

Article L.111-1 du Code de la propriété intellectuelle. 


\title{
La voie de l'a-féminité
}

\author{
Voix-off et subjectivité féminine dans The Man Who Envied Women \\ d'Yvonne Rainer
}

\author{
Johanna Renard
}

Dans son essai More Kicking and Screaming: from the Narrative Front/Backwater, Yvonne Rainer, chorégraphe et danseuse devenue cinéaste dans les années 1970, analyse ainsi sa trajectoire :

En partant des descriptions d'une expérience féminine individuelle débarrassée du contexte social et de la hiérarchie narrative, puis de celles de l'expérience individuelle féminine radicalement juxtaposée aux événements historiques, pour arriver à des spéculations nettement féministes sur l'expérience féminine, j'ai simplement formulé une évolution qui, en devenant plus explicitement féministe, semble exiger un ancrage plus solide dans les conventions narratives ${ }^{1}$

2 Elle fait référence à ce qu'elle a appelé son "dilemme narratif », une attirance mêlée de répulsion à l'égard de la narration dont elle s'était totalement débarrassée dans sa danse durant les années soixante puis avec laquelle elle avait peu à peu renoué en se tournant vers le cinéma au début des années 1970. De fait, le passage de Rainer de la danse au cinéma s'explique en partie par son intérêt croissant pour le traitement de l'émotion et des rapports humains et par le développement de sa conscience politique. Ainsi, si le refus de la narration est évident dans son premier film, Lives of Performers (1971), qui tient de la performance filmée et qui met en scène un trio amoureux, Journeys from Berlin/1971 (1981) marque l'alliance entre l'expérience personnelle et le contexte historico-politique en mettant en parallèle la psychothérapie d'une femme qui a tenté de se suicider, le journal intime d'une adolescente et une réflexion l'usage de la violence dans le combat politique. Enfin, The Man Who Envied Women $\left(1985^{2}\right)$, le cinquième film d'Yvonne Rainer, révèle clairement un besoin de réconciliation avec les conventions narratives, même si la narration demeure disjointe, elliptique et statique. Quoi qu'il en soit, c'est toujours à travers la voix-off que Rainer expérimente de nouvelles formes de narrations, donnant ainsi la primauté au texte sur l'image, dans la lignée d'Hollis Frampton. Et c'est dans TMWEW que le rôle formel et conceptuel de la voix-off est le plus marquant. En effet, inspirée par sa lecture des théories féministes du cinéma, Rainer propose une réflexion 
sur les rapports de genre et sur l'instrumentalisation du corps féminin en mettant en place des stratégies cinématographiques alternatives. Le recours à une voix-off féminine, une héroïne absente de l'image et supposément située hors champ, participe de sa démarche stratégique. Quelles sont les fonctions de cette voix-off dans le contexte d'une déconstruction du narratif et d'une remise en cause des effets idéologiques produits par l'appareil cinématographique?

\section{Un cinéma politique et stratégique}

3 Même si TMWEW n'est pas structuré autour d'une narration classique, le fil conducteur du film semble être les faits et gestes du héros, Jack Deller, durant les deux jours qui suivent le départ définitif de son épouse du foyer conjugal. On observe ce dernier dans ses déambulations quotidiennes: chez son psychanalyste, au téléphone avec des femmes, faisant du sport, délivrant un pontifiant séminaire universitaire, recevant une de ses étudiantes chez lui ou devisant de politique et de féminisme lors d'une soirée. Ainsi, le premier plan de TMWEW nous le présente assis, fumant avec désinvolture face à la caméra, dans un espace absolument neutre, au fond noir indéterminé. Deller s'adresse alors à un psychanalyste invisible, situé à gauche de l'écran. « Docteur, je vous dirai tout ce que vous voulez savoir sur ma vie sexuelle ", dit-il. Puis le fond noir se révèle être un écran, sur lequel sont projetés des extraits de films noirs, de mélodrames ou de films indépendants, qui n'entretiennent pas forcément de rapport évident avec les confessions du héros. Néanmoins, cela construit un espace psychanalytique appartenant au domaine du fantasme, révélateur de désirs et d'angoisses. Significativement, les confessions de Deller sont composées de fragments de lettres de Raymond Chandler ${ }^{3}$. Ces extraits se focalisent sur les femmes, développant un discours misogyne assez stéréotypé. Deller apparait comme un personnage très arrogant, se targuant « d'en savoir presque trop sur les femmes ». Tout en adoptant ce ton condescendant, il ne cesse de clamer son amour pour elles. Il oscille constamment entre deux visions stéréotypées: celle de la femme comme créature presque sacrée, qu'il faut respecter, et celle de l'objet sexuel dont il peut disposer à sa guise. Cependant, universitaire de gauche très cultivé, Deller manie les références théoriques avec aisance. En outre, il est très au fait des problèmes politiques contemporains. La plupart des idées qu'il présente sont particulièrement progressistes ; il revendique notamment des positions féministes qui ne cadrent pas avec la conception étriquée des rapports entre les sexes énoncée devant son analyste. Rainer traite à la fois le discours de Deller comme une source d'information valable et comme un jargon hermétique presque ridicule. À ce sujet, la scène la plus significative se déroule dans une salle de conférence où Jack Deller délivre un cours sur le langage, mêlant à la linguistique les analyses de Foucault et celles de la théorie psychanalytique. La parole du héros est manifestement dévaluée par la cinéaste ; traitée comme un bruit de fond, maltraitée par le brouhaha des étudiants, elle devient à plusieurs reprises quasiment incompréhensible, lorsque la bande sonore est accélérée. Deller semble donc représenter l'archétype du héros masculin menant l'action, qui, des deux membres du couple, est significativement le seul à apparaître à l'écran et à être nommé.

4 Néanmoins, le personnage de l'épouse, qui n'apparait jamais vraiment à l'image, est loin d'être secondaire. Voix narratrice off, cette dernière est jouée par la danseuse et chorégraphe américaine Trisha Brown. Cette figure essentielle de la postmodern dance, membre du Judson Dance Theater, a beaucoup dansé avec Yvonne Rainer durant les 
années 1960 et $1970^{4}$. De même, l'héroïne de TMWEW est une artiste, peut-être chorégraphe puisqu'elle parle de répétitions au début du film, et par ailleurs une activiste politique. Pour évoquer l'implication militante de Trisha, Rainer insère des images tournées alors qu'elle-même participait au collectif Artists' Call Against US Intervention in Central America en réaction contre l'intervention impérialiste de Reagan au Salvador et au Nicaragua, ou lorsqu'elle assistait aux audiences juridiques opposant des artistes aux minorités ethniques miséreuses du Lower East Side à New York pour l'obtention de logements dans un quartier sévèrement touché par la spéculation immobilière. Ceci est loin d'être anodin car Yvonne Rainer s'appuie sur de nombreux éléments tirés de sa propre expérience dans ses films et il demeure toujours une ambiguïté entre ce qui relève de la fiction et ce qui est autobiographique. Aussi le personnage de Trisha est-il totalement inspiré de la personnalité et du vécu de la réalisatrice ? Rien n'est moins certain. Si Rainer a pioché dans son expérience pour construire son héroïne, il n'en demeure pas moins qu'elle lui a, par exemple, donné une fille, qu'elle-même n'a jamais eue. De plus, si la voix-off confie ses états d'âmes aux spectateurs ou raconte ses déboires sentimentaux, elle relie toujours son expérience personnelle à une analyse plus large de la société, qui puise largement dans les discours féministes, marxistes et psychanalytiques. Outre sa vie sentimentale, elle évoque longuement la place des femmes dans la société, à travers, par exemple, l'impasse de relations hétérosexuelles où l'homme ne veut concéder aucun pouvoir, ou encore le tabou de la ménopause dans une société qui hystérise le corps des femmes. Très lucide, elle dresse un portrait sans concessions du héros comme de sa propre faiblesse. Contrairement à la voix masculine si assurée, l'héroïne commente, questionne, expose ses doutes mais n'impose jamais son point de vue. Si elle n'intervient pas à l'image, ce personnage n'est pas cantonné à un rôle traditionnel de narratrice off. Car il se passe autant de choses dans l'espace visuel que dans l'espace sonore. Ainsi, l'héroïne reçoit un appel téléphonique d'amis lui parlant du film d'Eustache, La Maman et la Putain (1973), ou l'interrogeant au sujet du nom de son héros, Jack Deller (et, une nouvelle fois, on se demande si Trisha n'est pas, en réalité, Yvonne). Par ailleurs, elle "reçoit" hors champ des individus, hommes et femmes - parmi lesquels l'artiste féministe Martha Rosler - qui commentent un collage présenté à l'image, dont elle est l'auteur, et qui est composé de coupures de presse, d'affiches et de photographies. Par ailleurs, on peut certes parler de narration, mais celle-ci est tout de même sérieusement mise à mal. Comme l'explique Yvonne Rainer :

The Man Who Envied Women se situe en dehors du cinéma narratif classique. Il n'y a pas d'intrigue [...] et, bien qu'il soit possible d'entendre la voix du narrateur en celle de l'héroïne (absente), cette voix fait une entorse aux conventions en refusant de faire avancer une histoire ou de défendre une thèse unique qui en nouerait les différents fils ${ }^{5}$

5 Pourquoi ce refus de la narration traditionnelle ? Il faut rappeler que le cinéma d'Yvonne Rainer est profondément politique et stratégique. La méfiance dont la cinéaste témoigne à l'égard du narratif doit être rapportée au projet commun de l'avant-garde cinématographique dans les années 1960 et 1970. Ainsi dans "The Two Avant-Garde ${ }^{6}$ ", Peter Wollen distingue deux tendances dans le cinéma d'avant-garde. Le premier mouvement, que Wollen appelle le «Co-op movement ", particulièrement développé aux États-Unis, est l'héritier des recherches formelles de l'art moderne. Il regroupe sous cette appellation des cinéastes comme Stan Brackhage ou Michael Snow qui, concevant un cinéma non narratif et abstrait, sont intéressés par le médium cinématographique en luimême (couleur, grain, montage, son, pellicule, etc.) et par de l'expérimentation pure. À 
l'opposé, Wollen met en évidence un cinéma avant-gardiste plutôt européen qui demeure attaché à la narration tout en remettant en cause ses conventions. Ainsi, Godard ou Straub et Huillet cherchent à se détacher des codes de l'idéologie dominante en proposant un travail sur le signifiant et le signifié. Le cinéma d'Yvonne Rainer est à rapprocher de ce second courant, car il se détourne d'un travail sur le médium, que Peter Gidal qualifie de matérialiste, pour offrir une place centrale au langage et à l'engagement politique.

Surtout, il s'agit d'un cinéma profondément lié aux recherches théoriques de son époque. Yvonne Rainer a été notamment extrêmement influencée par les théories féministes du cinéma, qu'elle découvre dès 1976. Pour TMWEW, elle confie "s'être littéralement appuyée sur la critique des films hollywoodiens de Laura Mulvey [...] dans son essai précurseur "Visual Pleasure and Narrative Cinema"" ". Faisant partie des textes fondateurs de la théorie féministe du cinéma, cet article s'appuie sur la psychanalyse et le marxisme pour dénoncer un dispositif cinématographique qui construit la femme comme objet, à la fois fascinant et menaçant, du regard masculin. Rappelons que, selon Mulvey, pour provoquer un plaisir scopique chez le spectateur, le cinéma hollywoodien sexualise à l'extrême le personnage féminin en l'offrant à la contemplation érotique. Élément indispensable du plaisir visuel, la femme représente une silhouette sur laquelle sont projetés les fantasmes masculins. Sa place est à part au sein du film car elle est exhibée à deux niveaux, «d'une part comme objet érotique pour les personnages de l'histoire et d'autre part pour les spectateurs dans la salle ${ }^{8} »$.

De surcroît, Rainer s'est aussi nourrie des écrits de Teresa de Lauretis qui, dans la lignée de Mulvey, dénonce le sexisme des structures narratives. Dans « Desire in Narrative " " la théoricienne explore la place de la femme au sein de la narration et souscrit au postulat de Mulvey selon lequel le récit génère le désir à travers l'usage de schémas sadiques masculins. Calqué sur une conception linéaire de l'existence humaine, le modèle de la narration est le périple du héros mythique qui, sur le chemin de la vie, est mis en difficulté par quelques obstacles qu'il doit surmonter pour atteindre son but. Le récit mythologique propose une distinction constante entre l'humain et le non-humain. Le rôle du héros est de détruire tout ce qui menace, par sa différence, le modèle instauré par l'homme (le monstre, la bête, le vilain, etc.). Dans ce schéma où il s'agit de distinguer un sujet et des obstacles, le récit construit la femme comme Autre. Prenant pour référence le mythe d'œEdipe, de Lauretis affirme :

Dire que le récit est le produit d'ÆEdipe, c'est affirmer que tout lecteur - homme ou femme - est contraint et défini par les deux positions d'une différence sexuelle ainsi conçue: l'homme-héros-humain du côté du sujet; et la femme-obstaclefrontière-espace [du côté de l'objet $\left.{ }^{10}\right]$.

8 Aussi, le désir dans le récit est forcément masculin. Cependant, Teresa de Lauretis n'ignore pas les efforts des cinéastes féminines pour faire émerger un désir féminin dans la narration. Cependant, comment les femmes peuvent-elles être attirées vers un dispositif qui les exclut? La solution ne serait-elle pas de se débarrasser totalement du récit et de suivre la voie tracée par le cinéma structuraliste? Mais dans ce cas comment créer un cinéma qui se fasse l'écho des combats politiques de son époque et plus particulièrement du féminisme? Or, on l'a vu, Rainer observe dans son propre travail une attirance grandissante pour la narration à mesure que sa conscience politique féministe s'affermit. Analysant l'évolution observable dans le travail de Rainer, De Lauretis parle d'une «stratégie de la cohérence ${ }^{11}$ ». Si un certain cinéma féministe peut nécessiter « un 
ancrage plus solide dans les conventions narratives ", c'est toutefois en les déconstruisant pour les subvertir. Pour de Lauretis :

Le travail cinématographique et féministe le plus passionnant aujourd'hui ne se construit pas contre le récit et l'œ®ipe ; c'est plutôt l'inverse. Il est doublement narratif et œdipien, en ce qu'il tente de souligner la duplicité du scénario et la contradiction spécifique constituée par le sujet féminin dans le récit ${ }^{12}$.

Se défiant de la structure temporelle illusionniste du cinéma narratif traditionnel, Rainer soumet son TMWEW à une structure prédéterminée non pas au service d'une recherche formelle mais d'une recherche théorique. Ainsi, en réaction aux théories féministes, Yvonne Rainer décide de développer des stratégies permettant de mettre à mal les rapports entre les sexes impliqués par le cinéma mainstream. De ce fait, elle joue avec les conventions narratives et propose des alternatives à la domination masculine. À première vue, il peut sembler que TMWEW - en donnant le rôle principal au membre masculin du couple, seul à apparaittre à l'écran - favorise la traditionnelle identification narcissique du spectateur au moi idéal constitué par le personnage principal. Cependant, Rainer sape ce processus d'identification en dédoublant son héros, qui est joué par deux acteurs différents : Larry Loonin et William Raymond, venant tous deux du théâtre expérimental. Par ailleurs, en supprimant la présence physique de Trisha, le but avoué de la réalisatrice est d'éviter toute réification du corps de la femme. Enfin, pour que la position de la femme dans le récit ne soit pas réduite au statut d'obstacle dans le parcours du héros, elle met en place « une voix extra-diégétique, une voix indépendante des personnages et de l'histoire, passablement active dans chaque scène ${ }^{13}$ »

\section{Désincarner la voix féminine}

10 La narration élaborée par Yvonne Rainer s'appuie, comme on l'a préalablement remarqué, sur le rôle essentiel de la voix-off. Cette dernière occupait déjà une place prédominante dans Journeys from Berlin/1971 à travers un couple échangeant des positions politiques, en off, dans un temps et un lieu différents des images montrées à l'écran, ou par le biais d'une adolescente invisible lisant son journal intime. Par le biais de la voix-off, la cinéaste rompt avec la connotation des images, créant des effets de rupture. Mais, afin d'analyser le rôle du personnage de Trisha dans TMWEW, il faut interroger cette notion. En effet, le terme de voix-off est-il le plus adapté pour décrire cette voix? Pour le déterminer, il faut tout d'abord parvenir à situer spatialement et temporellement le personnage. En effet, la voix de Trisha existe-t-elle dans le lieu et le temps de la situation montrée à l'écran (elle serait alors placée hors champ) ou bien appartient-elle à un autre temps et à un autre lieu que les images (il s'agirait alors d'une voix-off à proprement parler) ? Il n'est pas très aisé de répondre à cette question car Rainer s'attache à brouiller les pistes.

11 Selon la critique de cinéma Bérénice Reynaud la narratrice de TMWEW ne peut être assimilée à une traditionnelle voix-off : il s'agirait plutôt d'un personnage acousmêtre. En reprenant ce terme, elle se réfère à l'analyse de la voix au cinéma développée par Michel Chion ${ }^{14}$. Le théoricien donne le nom d'acousmêtre à toute voix dont l'émetteur n'a jamais été visualisé tout en constituant un personnage à part entière. Celui-ci se situe dans le même espace spatio-temporel que ce qui est visible mais parle d'un lieu en retrait de l'image, il demeure hors champ. De fait, la caractéristique principale de ce personnage invisible est d'être "présenté comme susceptible à tout moment d'apparaître dans le 
champ $\mathrm{p}^{15}$ ». Ainsi, ce dernier se définit finalement surtout par rapport aux limites du cadre. Selon Reynaud, cela définit le rôle du personnage de Trisha, toujours en passe d'apparaître à l'image : il s'agit d'une voix pas-encore-vue. Et elle en prend pour preuve plusieurs exemples. Ainsi, au début du film, lorsque l'héroïne déménage de l'appartement qu'elle occupait avec Jack, un corps féminin, qui demeure de dos, entre dans le cadre et échange quelques phrases banales avec l'homme quitté. Elle s'exprime alors avec la voix de Trisha Brown mais il n'y a aucune certitude que ce soit réellement le corps de la danseuse qui apparaît à l'écran. Par ailleurs, celui-ci apparaît plus tard dans le film mais dans l'espace fantasmatique de Deller, projeté derrière lui durant ses séances de psychanalyse. Pendant que ce dernier expose l'échec de son mariage et la récurrence de ses infidélités, on voit l'extrait d'un solo de Trisha Brown, Watermotor (1978), filmé par Babette Mangolte. Enfin, Reynaud démontre que l'héroïne est omniprésente à l'image, mais de manière indirecte, à travers notamment le collage qu'elle a laissé au mur de l'appartement de Deller et que ce dernier passe son temps à modifier. Cette œuvre fonctionne comme une trace physique obsédante de Trisha à l'image. Néanmoins, il faut remettre en cause l'assimilation de Trisha à un acousmêtre. En effet, même si Rainer entretient une grande ambiguïté et une certaine porosité entre les espaces visuels et acousmatiques, même si le personnage féminin peut sembler susceptible de se risquer dans l'image, il n'en demeure pas moins que sa voix paraît provenir d'un temps et d'un lieu différents de ce qui se passe à l'écran. Pour affiner la définition de cette voix, il faut se pencher de manière plus détaillée sur la fameuse scène de la pseudo-apparition de Trisha à l'image, citée par Reynaud. Ainsi, voici les faits que l'hérö̈ne énumère avant que ce que l'on présume être son corps ne surgisse :

Ce fut une semaine difficile. Alicia partit pour Antioch. Je quittai la maison de Jack pour mon studio. Je reçus une lettre d'expulsion. Je fêtai mon cinquantième anniversaire ${ }^{16}$.

12 Puis, une femme de dos, coiffée d'un chapeau et vêtue d'un imperméable, entre dans le cadre. On entend alors une voix que l'on lie tout naturellement au corps. «Jack, je pars maintenant. Je... Je passerai prendre le reste de mes affaires la semaine prochaine », ditelle. Ce dernier lui répond, de manière assez agressive, qu'il souhaiterait qu'elle lui précise le jour de son passage. La première chose importante à relever dans cette séquence, c'est que la voix narrative s'exprime au passé. La narration survient donc après les événements racontés, elle est ultérieure. Par ailleurs, le bref dialogue entre les deux membres du couple semble révélateur de la distance temporelle qui sépare l'acte narratif et les évènements racontés. En outre, Rainer révèle son stratagème dans le scénario. $\mathrm{Au}$ moment où le mystérieux corps apparait, la didascalie précise : « Nous supposons que la voix que nous entendons est la sienne. En réalité, sa voix est toujours off ${ }^{17} »$. La voix de Trisha n'est donc clairement pas située hors champ puisqu'il y a une non-coïncidence entre le corps et la voix. La femme à l'image est une projection du passé que la narratrice nous donne à voir. On peut en conclure que le personnage est totalement extérieur à l'image. Il possède les caractéristiques principales de la voix-off: il se situe dans un espace-temps différent et parle après les événements montrés à l'écran, qu'il analyse et décortique pour les spectateurs. Or, l'usage de ce type de voix, qui plus est féminine, dans un film qui affirme son dialogue avec les théories féministes, est particulièrement significatif.

13 Alain Boillat l'a évoqué dans son étude des narrateurs over : « On peut dire que la position du sujet masculin est assurée de façon optimale par la "présence-absence" qui définit l'origine discursive d'un texte over, puisqu'elle permet d'allier l'exercice d'un pouvoir 
- celui du verbe dont est censée découler l'image - et l'éviction du régime du visible ${ }^{18}$ ». Dans TMWEW, la voix-off féminine s'oppose au discours envahissant de Jack Deller. L'omniprésence et l'importance de ce dernier sont illusoires: c'est l'héroïne qui commente, qui analyse ou qui interrompt le discours masculin. De ce fait, elle obtient le pouvoir traditionnellement conféré au narrateur omniscient. Ainsi, Trisha voit tout, entend tout et semble être partout. Elle sait par avance ce qui va se passer, ce qui va être dit. Surtout, elle décrypte de manière très fine les propos du héros, qu'elle semble connaître par cœur. Elle interrompt par exemple les confidences de Deller à son analyste pour prévenir les spectateurs: "C'est là où il dit "un homme n'est rien sans une femme" ». Rainer semble signifier que c'est en échappant au regard spectatoriel que la voix féminine conquiert une légitimité. En effet, dans l'imaginaire cinématographique, la voix-off se voit traditionnellement attribuer les pouvoirs d'ubiquité, de panoptisme, d'omniscience et d'omnipotence. De ce fait, au cinéma, ces voix sont majoritairement masculines. À ce sujet, Kaja Silverman a apporté une contribution essentielle à la théorie féministe du cinéma qui, dans le sillage de Mulvey, avait concentré son intérêt sur l'image de la femme. Elle montre que la voix joue un rôle crucial dans la représentation de la féminité au cinéma. En cantonnant les femmes au champ visible, le cinéma « classique » a pour pratique d'enchaîner, d'ancrer les voix féminines au corps. En ce sens, la synchronisation permet de dénier tout pouvoir aux femmes. Elle véhicule la conception d'une féminité charnelle, sexuelle, esclave des passions et des émotions à laquelle on peut de ce fait dénier toute autorité. Ainsi, la voix des actrices se doit d'être sensuelle et érotique (pensons à Marlène Dietrich, Greta Garbo ou Lauren Bacall) pour accentuer la sexualisation du corps. La différence sexuelle est construite par la dévalorisation constante de la voix, et donc de la parole, féminine. L'usage de la voix-off dans TMWEW est à la fois un outil formel et conceptuel: il contribue à la remise en cause de l'appareil cinématographique mais représente aussi une réflexion sur la représentation des femmes en tant qu'image et en tant que sujet. Il s'agit d'une «stratégie de la cohérence » qui permet de tirer profit de la narration en modifiant les règles de la représentation du sujet sexué et en imposant le désir féminin.

Mais comment représenter la subjectivité féminine ? La réponse d'Yvonne Rainer semble être : en mettant à mal les codes de la représentation cinématographique. Il s'agit là d'une posture adoptée par de nombreuses cinéastes femmes, influencées par le mouvement féministe, qui choisissent de détruire ou de subvertir les conventions de la synchronisation. Dans "Dis-embodying the Female Voice ", Silverman démontre qu'il s'agit là de libérer la parole de la femme de son corps pour laisser entendre la subjectivité féminine ${ }^{19}$. Finalement, le discours, plus que l'image, est un enjeu essentiel pour ce type de cinéma. Selon Silverman, il est un outil capital dans la remise en cause de l'appareil cinématographique patriarcal. Privilégié, l'usage de la voix-off permet d'offrir une place à la confession et à l'introspection, en se basant souvent sur l'expérience autobiographique (India Song de Marguerite Duras ou Je, tu, il, elle de Chantal Akerman). Ainsi, il n'est pas anodin que la voix narratrice de TMWEW soit à la première personne. De ce fait, elle semble s'adresser à chacun de nous de manière intime. Pivot de l'identification, particulièrement englobante, elle résonne en chaque spectateur ou spectatrice comme sa propre voix. De surcroit, Trisha est, on l'a vu, fortement impliquée dans l'image. Selon Chion, il y a deux types de voix-off: une voix qui demeure extérieure aux spectateurs, placée en retrait, et ce qu'il appelle la voix-je. Cela définit une voix non filtrée, non réverbérée, non manipulée techniquement qui absorbe le spectateur ou la spectatrice du fait de sa grande proximité. Généralement, cette voix se positionne en marge de la 
diégèse visualisée mais s'engage résolument dans l'image. Le personnage de Trisha, qui possède une position énonciatrice très forte, relève clairement de la voix-je. Cependant, analysant ce type de voix-off, Christian Metz observe : «Aussi longtemps qu'elle parle et demeure invisible, elle bouche de son corps absent l'accès à la Voix du film, se substitue à elle et se confond avec ce que, pourtant, elle n'est pas, le point d'origine de la narration ${ }^{20}$ ». Or, il serait erroné d'identifier Trisha à la voix du film car elle ne prend pas du tout en charge son organisation. D'ailleurs, à la différence des voix-off classiques, elle n'est pas dotée d'un savoir illimité et n'est certainement pas toute-puissante. Contrairement à la voix masculine si assurée, l'héroïne commente, questionne, tâtonne mais n'impose jamais son point de vue. Au contraire, elle ne cesse de douter, de se tromper parfois, de remettre en cause ce qu'elle sait et ce qu'elle voit. La cinéaste a consciemment voulu se prémunir de ce qu'elle appelle le "syndrome de la voix du maître ». Durant toute sa carrière de chorégraphe comme de cinéaste Rainer s'est radicalement opposée aux procédés visant à fasciner et à manipuler le spectateur. Dans TMWEW, cela passe par la confrontation de voix d'auteurs différentes, parfois contradictoires. En effet, l'esthétique filmique de Rainer est principalement basée sur le collage d'éléments disparates. Elle qualifie ellemême sa stratégie esthétique de "brigandage " de la narration. Ainsi, dans la trame narrative de TMWEW sont insérés des éléments extra-diégétiques qui peuvent être des extraits de discours politiques, de textes théoriques (Foucault, Jameson, Kristeva, Lacan), des extraits de films, des bribes de conversations ou des images de manifestions. Tous ces éléments ont été consciemment "empruntés " à droite à gauche et soumis à des juxtapositions quelque peu radicales. Même si le terme de brigandage peut paraître exagéré, il y a certainement une forme de violence contestataire et facétieuse dans les distorsions imposées par Rainer à la narration et aux conventions cinématographiques. Par ailleurs, ce «brigandage » pose la question de l'autorité et de l'auteurité au féminin. Ainsi, Rainer a pris acte de la mort de l'auteur annoncée par Foucault et Barthes, qui n'est pas sans poser problème pour les femmes car la légitimité associée au statut d'auteur leur a été longtemps refusée. Aussi, les femmes doivent-elles ressusciter cette voix qu'elles n'ont pas pu adopter ou bien contribuer à sa destruction? En créant une œuvre polyphonique qui procède par emprunt et par collage, Rainer choisit, elle, de ne pas reconstruire la voix de l'auteur, et ce même dans un but féministe.

\section{Un espace off « ironique »}

15 En nous penchant sur la question de la voix-off, nous avons mis en évidence la difficulté de définir l'espace acousmatique dans ce film. Lorsque la source du son est invisible, on se demande constamment si celui-ci est situé hors-champ ou bien dans l'espace off. Évoquons par exemple une scène étrange qui met à mal les éventuelles certitudes des spectateurs (s'il leur en reste encore à ce stade du film). Elle montre le héros déambulant dans son appartement, dans lequel il est de toute évidence seul, puis s'asseyant pour lire Playboy. Tout à coup, la caméra montre en gros plan des jambes surgissant du côté gauche. Une femme se penche afin que son visage apparaisse à l'écran. Ses lèvres sont peintes de rouge vif. Elle énonce, en articulant exagérément: "Est-ce que toutes les femmes ayant leurs règles peuvent quitter la salle s'il vous plaît?». Dans le scénario, celle-ci est simplement identifiée comme "une femme» mais les spectateurs peuvent aisément reconnaître Yvonne Rainer. Cette intervention représente l'une des nombreuses ruptures dans la narration et pose de nombreuses questions. On se demande notamment 
de quel espace non visualisé cette femme surgit-elle. Mais on s'interroge aussi sur la présence de la cinéaste dans son propre film, qui met à mal la frontière entre réalité et fiction. De même, il y a plusieurs séquences où des locuteurs invisibles commentent un collage montré à l'écran. On se demande alors si ces derniers sont placés hors champ, devant le collage réel, ou bien s'ils regardent une projection du film, comme les spectateurs. Le rapport espace visuel - espace acousmatique se complexifie lorsqu'Yvonne Rainer s'entretient dans l'espace sonore avec Martha Rosler au sujet de cette « œuvre », pendant que nous voyons à l'image Jack Deller en modifier l'agencement. Ces interventions instaurent une troublante indétermination spatio-temporelle. Car même lorsqu'elle intervient dans l'image, dans la séquence du Playboy, Rainer ne semble pas se situer dans le même temps que le héros. Elle ne peut être définie ni entièrement comme une voix-off, ni totalement comme un acousmêtre. De ce fait, les spectateurs ne peuvent déterminer, ou très difficilement, si l'espace sonore est hors-champ ou off. Michel Chion évoque longuement ce type d'indétermination présent dans India Song (1974) de Marguerite Duras. Dans ce film, la réalisatrice supprime presque totalement le son in en faisant largement entendre du son hors champ (la voix des protagonistes qui sont sortis du cadre, l'orchestre invisible du bal de la réception) ou off (des voix parlant au passé des personnages sur l'écran). On ne sait jamais dans quel temps se situent ces sons : dans le présent (le hors champ) ou dans un autre temps (le off) ? Selon Chion, « le charme de ce film vient précisément de cette porosité, de cette indétermination de la cloison qui sépare les deux espaces acousmatiques ${ }^{21}$ ». Dans TMWEW, l'ancrage spatio-temporel est constamment miné et cela crée une incertitude très productive. Comme l'analyse Chion, au cinéma «la zone acousmatique se définit comme fluctuante, susceptible en permanence d'être remise en cause par ce que l'on va voir ${ }^{22}$ ». Or, dans le film de Rainer, il y a toujours un risque que le off perturbe le cadre. D'ailleurs, cette zone acousmatique est particulièrement active: on y débat, on s'y interpelle, on s'y révolte. Il s'agit d'un véritable univers parallèle aux images, où il se passe parfois davantage de choses qu'à l'écran.

16 Au fond, le film nous donne l'impression d'être placés aux côtés de Trisha, de débattre avec les «experts» décryptant le collage. Il semble presque que ces personnages commentant les images qui défilent sur l'écran sont assis dans la salle de cinéma avec les spectateurs, donc dans le même espace spatio-temporel que ces derniers. Cette hypothèse s'impose lorsque le film montre une salle de cinéma dans laquelle est projeté un extrait de Night of the Living Dead (1968) de George Romero. La caméra est placée derrière les spectateurs, nous donnant l'illusion d'être installés dans la salle avec eux. Puis, imitant ce qu'il voit à l'écran, le public entre en furie et commence à se battre. On se demande alors, encore une fois, quelle est la frontière entre la fiction et la réalité et surtout, quelle est la limite de l'espace spectatoriel.

17 Analysant la voix-off, le critique de cinéma Serge Daney affirme que cette voix parallèle aux images «ne s'adresse (toute entière, sa face énoncé et sa face énonciation) qu'au spectateur avec lequel elle fait alliance ${ }^{23} »$. On ressent fortement cette alliance avec les spectateurs dans TMWEW. Non plus fascinés ou manipulés, le film leur donne la possibilité de débattre des images qui défilent sur l'écran, de questionner les discours présentés. Cette impression de pouvoir intervenir dans le film est accentuée par les interventions qui se déroulent dans l'espace off (le coup de téléphone d'amis de Trisha qui lui posent les questions que les spectateurs se posent, l'interview de Jack Deller par Trisha, ou celui de Martha Rosler par Yvonne Rainer). La cinéaste poursuit ici une recherche amorcée dans 
ses pièces chorégraphiques qui vise à interroger le statut des spectateurs en refusant leur subordination, en leur offrant la possibilité de conserver indépendance et esprit critique face au spectacle qui leur est présenté. Ainsi, aucun discours n'est favorisé par rapport à un autre, tout est sujet à critique et à contradiction. D'ailleurs, en révélant que les stratégies mises en place par Rainer dans TMWEW sont explicitement des réponses aux débats émergeant dans les théories féministes du cinéma, il ne faudrait pas commettre l'erreur de réduire le cinéma de Rainer à un objectif purement illustratif ou didactique. À mille lieux de cela, ce dernier est d'une telle ambiguité qu'il peut même en paraître insaisissable et énigmatique.

De surcroît, en remettant en cause un appareil cinématographique qui dénie aux femmes le statut de sujet, l'un des buts avoués de Rainer est de remettre en cause l'homogénéité sexuelle du spectateur. En effet, durant les années 1980, la théorie filmique féministe s'interroge sur la place de la spectatrice au cinéma, montrant que le cinéma s'adresse généralement au spectateur masculin en répondant à ses désirs. Certaines théoriciennes comme Laura Mulvey affirment que, pour éprouver du plaisir en tant que spectatrices, les femmes seraient contraintes de s'approprier le regard masculin ${ }^{24}$. De ce fait, leur désir et leur subjectivité seraient reniés, refoulés, censurés. Dans TMWEW, Rainer pose la question du désir féminin en créant cet espace off et en permettant ainsi à l'héroïne d'accéder à un véritable statut de sujet. Il s'agit d'un espace de liberté qui n'a rien de fictionnel, qui semble se confondre avec le réel même si cela ne peut demeurer qu'illusoire en raison des caractéristiques du médium cinématographique. Il n'empêche que Rainer crée aussi un autre type de spectatoriat. Ainsi, TMWEW remet en cause la notion de spectateur de référence en apparence non sexué mais qui est, en fait, masculin. Comme le montre De Lauretis, "en déconstruisant l'espace narratif, le film construit un espace critique dans lequel [la spectatrice] est interpellée, précisément, comme femme et comme a-femme ${ }^{25}$ ». Cette notion d'a-féminité apparaît à la fin du film quand la voix de la narratrice confie :

Je ne peux vivre sans hommes, mais je peux vivre sans un homme. Cette pensée m'est déjà venue, mais cette fois l'idée n'est pas teintée de douleur, de désespoir ou de fatalité. Je sais que la tristesse sera parfois atroce. Mais je sais aussi qu'il y a désormais quelque chose de différent. Quelque chose qui s'apparente à l'inféminité. Pas une femme nouvelle, pas une non-femme ou une misanthrope ou une antifemme, et pas une lesbienne non pratiquante. Infemme est peut-être aussi un mauvais terme. A-femme est plus exact. A-féminin. A-féminité26.

19 En construisant une subjectivité féminine à travers un personnage sans corps, Rainer ne s'atèle pas seulement à une remise en question des stéréotypes de genre, elle interroge les constructions de la masculinité et de la féminité dans la société contemporaine et s'interroge sur la possibilité de construire une identité autre, non plus déterminée par le sexe biologique.

On l'a vu, TMWEW est un film profondément politique sinon militant. Voix-off et narration sont autant d'armes stratégiques mises en place par Yvonne Rainer pour subvertir l'idéologie patriarcale imposée par l'appareil cinématographique. Cependant, ne nous trompons pas, l'artiste s'est toujours méfiée du didactisme et des dogmes théoriques. Aussi, dans TMWEW comme dans ses autres films, tout discours est sans cesse attaqué, nuancé, remis en question. En créant un espace acousmatique où son héroïne s'exprime en toute liberté, Yvonne Rainer donne surtout la part belle à l'ambiguïté, qu'elle soit poétique (la confusion spatio-temporelle, la porosité entre les espaces) ou sexuelle. Toutefois, on peut reprocher à la stratégie développée par Rainer de faire montre d'un certain puritanisme. En effet, elle efface purement et simplement le corps 
féminin pour lutter contre la domination patriarcale. Certes, il y a eu quelques précédents. On pense au travail du cinéaste anglais Peter Gidal, qui refuse de faire apparaitre toute image de femme dans ses films des années 1970 car il ne voit pas comment «ces images peuvent être séparées des significations dominantes ${ }^{27}$ ». Rainer revendique aussi l'influence du film de Godard et Miéville Comment ça va (1978), dans lequel le visage de l'héroïne est toujours dans l'ombre ce qui lui confère un pouvoir qu'elle n'aurait pas si elle apparaissait entièrement à l'image. Mais cette solution est quelque peu extrême en ce qu'elle représente une négation du corps, réduisant la subjectivité féminine à l'intellect. Or, le corps dissimulé est celui d'une danseuse et chorégraphe, Trisha Brown, pour laquelle il représente l'instrument de travail premier. Le refus du corps qui semble se manifester dans TMWEW parait entrer en totale contradiction avec le travail chorégraphique de Rainer et avec ses premiers films, qui offrent une place centrale au corps et aux exercices chorégraphiques. Sans doute, TMWEW représente la réponse la plus radicale au NO Manifesto de Rainer ${ }^{28}$ : ayant constamment lutté contre le narcissisme en danse, elle parvient au bout de cette logique en supprimant de l'image le corps de la danseuse.

\section{NOTES}

1. Yvonne Rainer, "Plus de coups et de cris du front/arrière de la narration ", [1982], in Une femme qui...Écrits, entretiens, essais critiques, Dijon, Les Presses du réel, 2008, p. 147

2. Dans la suite de ce texte, le titre The Man Who Envied Women sera abrégé en TMWEW.

3. Raymond Chandler (1888-1959), l'écrivain américain de romans noirs, a aussi travaillé comme scénariste pour Hollywood. Son roman Big Sleep a été porté à l'écran par Howard Hawks en 1946, tout comme Lady in the Lake, adapté par Robert Montgomery en 1946. Chandler a aussi travaillé à l'adaptation de Double Indemnity de James M. Cain pour Billy Wilder en 1944.

4. Trisha Brown a dansé dans plusieurs des pièces chorégraphiques les plus significatives de la carrière de Rainer. Citons Satie for Two (1962), Terrain (1963) ou Grand Union Dreams (1971).

5. Yvonne Rainer, «Ruminations sur de possibles antidotes cinématographiques au nœud de vipères œdipien », trad. Bérénice Reynaud, CinémAction, n 67, 1993, p. 178.

6. Peter Wollen, "The Two Avant-Gardes," Studio International vol. 190, $\mathrm{n}^{\circ} 978$ (Novembre/ Decembe 1975), pp. 171-175

7. Yvonne Rainer, Feelings are Facts. A Life, Cambridge, MIT Press, 2006, p. 456.

8. Laura Mulvey, «Visual Pleasure and Narrative Cinema » [1975], in Visual and Other Pleasures, Basingstoke, Macmillan, 1989, p. 18.

9. Teresa de Lauretis, "Desire in Narrative" in Alice Doesn't: Feminism, Semiotics, Cinema, Bloomington, Indiana University Press, 1984, pp. 103-157.

10. Teresa de Lauretis, « Desire in Narrative », art. cit., p. 121.

11. Teresa de Lauretis, «Strategies of Coherence. Narrative Cinema, Feminist Poetics, and Yvonne Rainer » in Technologies of Gender : Essays on Theory, Film, and Fiction, Bloomington, Indiana University Press, 1987, pp. 107-124.

12. Teresa de Lauretis, « Desire in Narrative », art. cit., p. 157.

13. Yvonne Rainer, «Réflexions sur le cinéma féminin : ravaler ses mots, exprimer ses luttes» [1986], in Une femme qui..., op. cit., p. 174. 
14. Bérénice Reynaud, «Impossible Projections », Screen, n² 28, vol. 4, automne 1987, pp. 40-53.

15. Michel Chion, Un art sonore, le cinéma: histoire, esthétique, poétique, Paris, Cahiers du cinéma, 2003, p. 411.

16. Yvonne Rainer, "The Man Who Envied Women. Film Script", in The Films of Yvonne Rainer, Bloomington, Indiana University Press, 1989, p. 177.

17. Ibid.

18. Alain Boillat, «Des personnages érigés en narrateurs: les voix over chez Joseph L. Mankiewicz (A Letter to Three Wives, All about Eve et The Barefoot Contessa) », Cahiers de narratologie [En ligne], n²0, 2011, mis en ligne le 26 août 2011, consulté le 27 février 2012.

19. Kaja Silverman, «Dis-embodying the Female Voice» in Mary Ann Doane, Patricia Mellencamp, Linda Williams (dirs.), Re-Vision: Essays in Feminist Film Criticism, Los Angeles, University Publications of America,1984, pp. 131-147

20. Christian Metz, L'énonciation impersonnelle ou le site du film, Paris, Méridiens Klincksieck, p. 141.

21. Michel Chion, Un art sonore, le cinéma, op. cit., p. 232.

22. Michel Chion, La voix au cinéma, Paris, Éditions de l'Étoile, p. 27.

23. Serge Daney, "L'Orgue et l'aspirateur. (La voix off et quelques autres) », Cahiers du cinéma, $\mathrm{n}$ - 278-79, août-septembre 1977, p. 24.

24. Voir notamment Laura Mulvey, «Afterthoughts on 'Visual Pleasure and Narrative Cinema' Inspired by King Vidor's Duel in the Sun », Framework, vol. 15-17, été 1981, pp. 12-15.

25. Teresa de Lauretis, «Strategies of Coherence. Narrative Cinema, Feminist Poetics, and Yvonne Rainer ", art.cit., p. 124.

26. «I can't live without men, but I can live without a man. I've had this thought before, but this time, the idea is not colored by stigma or despair or finality. I know there will sometimes be excruciating sadness. But I also know something is different now. Something in the direction of unwomanliness. Not a new woman, not a non-woman or misanthropist or anti-woman, and not non-practising lesbian. Maybe unwoman is also the wrong term. A-woman is closer. A-womanly. A-womanliness ».

27. Teresa de Lauretis, Stephen Heath (dirs.), The Cinematic Apparatus, New York, Macmillan, 1980, p. 169.

28. En 1965, en conclusion d'un article relatant le processus de création à l'origine de sa pièce Parts of Some Sextets, Yvonne Rainer publie son fameux "No Manifesto", texte lapidaire énumérant tous les oripeaux dont la danse doit, selon elle, se débarrasser : «NON au grand spectacle non à la virtuosité non aux transformations et à la magie et au faire-semblant, non au glamour et à la transcendance de l'image de la vedette non à l'héroïque non à l'anti-héroïque non à la camelote visuelle non à l'implication de l'exécutant ou du spectateur non au style non au kitsch non à la séduction du spectateur par les ruses du danseur non à l'excentricité non au fait d'émouvoir ou d'être ému» (Yvonne Rainer, "Some Retrospective Notes on a Dance for 10 People and 12 Mattresses Called Parts of Some Sextets », The Tulane Drama Review, vol. 10, $\mathrm{n}^{\circ}$ 2, hiver 1965, p. 178). 


\section{RÉSUMÉS}

Danseuse, chorégraphe puis cinéaste expérimentale, l'artiste américaine Yvonne Rainer a marqué l'histoire de l'avant-garde new-yorkaise des années 1960 aux années 1990. Son cinéma donne la primauté à la parole sur l'image, délaissant la narration classique au profit d'une juxtaposition de discours multiples. Si la voix-off tient une place centrale dans la plupart des films de Rainer, c'est dans The Man Who Envied Women (1985) que son rôle est le plus fondamental. En effet, influencée par la théorie féministe du cinéma et notamment par la réflexion de Laura Mulvey - qui montre comment le dispositif cinématographique construit la femme comme objet du regard masculin, à la fois fascinant et menaçant - Yvonne Rainer propose une réflexion sur les rapports de genre et sur l'instrumentalisation du corps féminin. Désirant contrer la fascination cinématographique pour l'image des femmes, elle transforme son héroïne en voix désincarnée, en personnage hors champ n'apparaissant jamais à l'image. Afin d'analyser comment le recours à un personnage principal invisible dans The Man who Envied Women permet à Rainer d'expérimenter de nouvelles formes narratives, on se penchera sur l'effet de cette voix-off en termes d'énonciation, de représentation, d'identification et d'auteurité.

\section{INDEX}

Index géographique : États-Unis

Mots-clés : cinéma expérimental, danse, féminisme, voix-off

Index chronologique : XXème siècle

\section{AUTEUR}

\section{JOHANNA RENARD}

Université de Rennes 2 (EA 1279). Histoire et critique des arts, doctorante en histoire de l'art contemporain 